\title{
Weak monotone rearrangement on the line*
}

\author{
Julio Backhoff-Veraguas ${ }^{\dagger} \quad$ Mathias Beiglböck ${ }^{\ddagger} \quad$ Gudmund Pammer $^{\S}$
}

\begin{abstract}
Weak optimal transport has been recently introduced by Gozlan et al. The original motivation stems from the theory of geometric inequalities; further applications concern numerics of martingale optimal transport and stability in mathematical finance.

In this note we provide a complete geometric characterization of the weak version of the classical monotone rearrangement between measures on the real line, complementing earlier results of Alfonsi, Corbetta, and Jourdain.
\end{abstract}

Keywords: convex order; mass transport; martingale transport; monotone rearrangement. AMS MSC 2010: 60E15; 60G42; 60J05.

Submitted to ECP on March 5, 2019, final version accepted on January 22, 2020.

\section{Introduction}

Recently, there has been a growing interest in weak transport problems as introduced by Gozlan, Roberto, Samson and Tetali [14]. While the original motivation mainly stems from applications to geometric inequalities (cf. Marton [16, 15] and Talagrand [21, 22]), weak transport problems appear also in a number of further topics, including martingale transport $[2,3,9,5]$, the causal transport problem [6, 1], and stability in mathematical finance [4].

\subsection{Framework and main results}

Write $\Pi(\mu, \nu)$ for the set of couplings between $\mu, \nu \in \mathcal{P}\left(\mathbb{R}^{d}\right)$. Starting with the seminal article of Gangbo-McCann [11] problems of the form

$$
W_{\theta}(\mu, \nu):=\inf _{\pi \in \Pi(\mu, \nu)} \int_{\mathbb{R} \times \mathbb{R}} \theta(x-y) \pi(d x, d y),
$$

where $\theta: \mathbb{R}^{d} \rightarrow \mathbb{R}$ denotes a convex function have received particular attention in optimal transport. A related problem that is considered in the weak transport literature is

$$
V_{\theta}(\mu, \nu):=\inf _{\mu^{*} \leq{ }_{c} \nu} W_{\theta}\left(\mu, \mu^{*}\right) .
$$

Here $\leq_{c}$ denotes convex order, i.e. $\mu \leq_{c} \nu$ iff $\int \varphi d \mu \leq \int \varphi d \nu$ for all convex $\varphi: \mathbb{R}^{d} \rightarrow \mathbb{R}$.

\footnotetext{
${ }^{*}$ MB gratefully acknowledges support by FWF-Grant Y00782. GP acknowledges support from the AustrianScience Fund (FWF) through Grant No. W 1245. All authors thank an anonymous referee for insightful comments that lead to a significant improvement of the article.

${ }^{\dagger}$ University of Vienna, Austria, and University of Twente, Netherlands. E-mail: julio. backhoff@utwente.nl

${ }^{\ddagger}$ University of Vienna, Austria. E-mail: mathias . beiglboeck@univie.ac .at

${ }^{\S}$ University of Vienna, Austria. E-mail: gudmund . pammer@univie.ac.at
} 
The problem (1.2), and in particular its one dimensional version, is investigated in $[2,14,13,19,17,20,10,12,7,4]$. The main purpose of this note is to give a complete geometric characterization of the optimizer $\mu^{*}$ in one dimension.

We write $\mathcal{P}_{\rho}\left(\mathbb{R}^{d}\right)$ for the set of probability measures on $\mathbb{R}^{d}$ which have finite $\rho$-moment.

Definition 1.1. Fix $\mu, \nu \in \mathcal{P}_{1}(\mathbb{R})$. We call a function $S: \mathbb{R} \rightarrow \mathbb{R}$ admissible if it satisfies

(i) $S$ is increasing,

(ii) $S$ is 1-Lipschitz,

(iii) $S(\mu) \leq_{c} \nu$.

Theorem 1.2. Let $\mu, \nu \in \mathcal{P}_{1}(\mathbb{R})$. There exists an admissible $T$ ( $\mu$-a.s. unique) which is maximal in the sense that $S(\mu) \leq_{c} T(\mu)$ for every other admissible $S$.

If $\theta: \mathbb{R} \rightarrow \mathbb{R}$ is convex then $\mu^{*}:=T(\mu)$ is an optimizer of (1.2). If $\theta: \mathbb{R} \rightarrow \mathbb{R}$ is strictly convex and $V_{\theta}(\mu, \nu)$ is finite, $T(\mu)$ is the unique optimizer of (1.2).

We call the ( $\mu$-a.s. unique) map $T$ in Theorem 1.2 the weak monotone rearrangement.

A consequence of Theorem 1.2 is that the optimizer of (1.2) does not depend on the choice of the convex function $\theta$. We find this fact non-trivial as well as remarkable and highlight that it is not new: different independent proofs were given by Gozlan, Roberto, Samson and Tetali [14], Alfonsi, Corbetta, Jourdain [2] and Shu [20]. For $d>1$ the optimizer of (1.2) may depend on $\theta$, see [2, Example 2.4].

The map $T$ can be explicitly characterized in geometric terms using the notion of irreducibility introduced in [8]: Measures $\eta, \nu \in P_{1}(\mathbb{R})$ are in convex order iff their potential functions satisfy

$$
u_{\eta}(y):=\int_{\mathbb{R}}|x-y| \eta(d x) \leq \int_{\mathbb{R}}|x-y| \nu(d x)=: u_{\nu}(y)
$$

By continuity, the set $U$ where this inequality is strict is open. Hence $U=\bigcup_{n} I_{n}$, where $\left(I_{n}\right)$ is an at most countable family of disjoint open intervals; these intervals $I_{n}$ are called irreducible with respect to $(\eta, \nu)$.

Theorem 1.3. The weak monotone rearrangement $T$ of $\mu, \nu \in \mathcal{P}_{1}(\mathbb{R})$ is the unique admissible map which has slope 1 on each interval $T^{-1}(I)$, where $I$ is irreducible w.r.t. $(T(\mu), \nu)$.

We emphasize that [2, Proposition 4.3] yields that every optimiser $\mu^{*}$ of (1.2) is of the form $T(\mu)$ for an admissible map $T$ and thus implies a part of Theorem 1.2. Likewise, it can be seen from [2, Proposition 4.3] that this mapping $T$ has slope 1 on each interval $T^{-1}(I), I$ irreducible w.r.t. $(T(\mu), \nu)$. The novelty of Theorem 1.3 is that there exists only one admissible mapping with this irreducibility property.

\subsection{Connection with martingale transport plans}

Intuitively, the irreducible intervals of $(\eta, \nu)$ are the components where we need to 'expand' $\eta$ in order to transform it into $\nu$. In this sense Theorem 1.3 asserts that the mass of $\mu$ can either concentrate between $\mu$ and $T(\mu)$, or it can expanded between $T(\mu)$ and $\nu$ (see Figure 1).

To make this precise, we recall from [14] that (1.2) can be reformulated as

$$
V_{\theta}(\mu, \nu)=\inf _{\pi \in \Pi(\mu, \nu)} \int_{\mathbb{R}^{d}} \theta\left(x-\int_{\mathbb{R}^{d}} y \pi_{x}(d y)\right) \mu(d x),
$$

where $\left(\pi_{x}\right)_{x \in \mathbb{R}^{d}}$ denotes a regular disintegration of coupling $\pi$ w.r.t. its first marginal $\mu$.

The set of optimizers of (1.4) is also straightforward to express in terms of $T$ : Write $\Pi_{M}(\eta, \nu)$ for the set of martingale couplings (or martingale transport plans), i.e. $\pi \in$ 
$\Pi(\eta, \nu)$ which satisfy barycenter $\left(\pi_{x}\right)=x, \eta$-a.s. By Strassen's theorem $\Pi_{M}(\eta, \nu)$ is nonempty iff $\eta \leq_{c} \nu$. Using this notation, $\pi \in \Pi(\mu, \nu)$ is optimal iff there exists a martingale coupling $\pi^{M} \in \Pi_{M}(T(\mu), \nu)$ such that $\pi$ is the concatenation of the transports described by $T$ and $\pi^{M}$ :

$$
\pi(A \times B)=\int_{A} \mu(d x) \pi_{T(x)}^{M}(B) .
$$

Any $\pi^{M} \in \Pi_{M}(\eta, \nu)$ can be decomposed based on the family of irreducible intervals $\left(I_{n}\right)_{n}$ : denoting $F:=\left(\cup_{n} I_{n}\right)^{c}$ by [8, Appendix A] we have

$$
\pi^{M}=\sum_{n} \pi_{\mid I_{n} \times \bar{I}_{n}}^{M}+(\mathrm{Id}, \mathrm{Id})\left(\mu_{\mid F}\right) .
$$

Plainly, (1.6) asserts that any martingale transport plan can move mass only within the individual irreducible intervals, whereas particles $x \in F$ have to stay put.
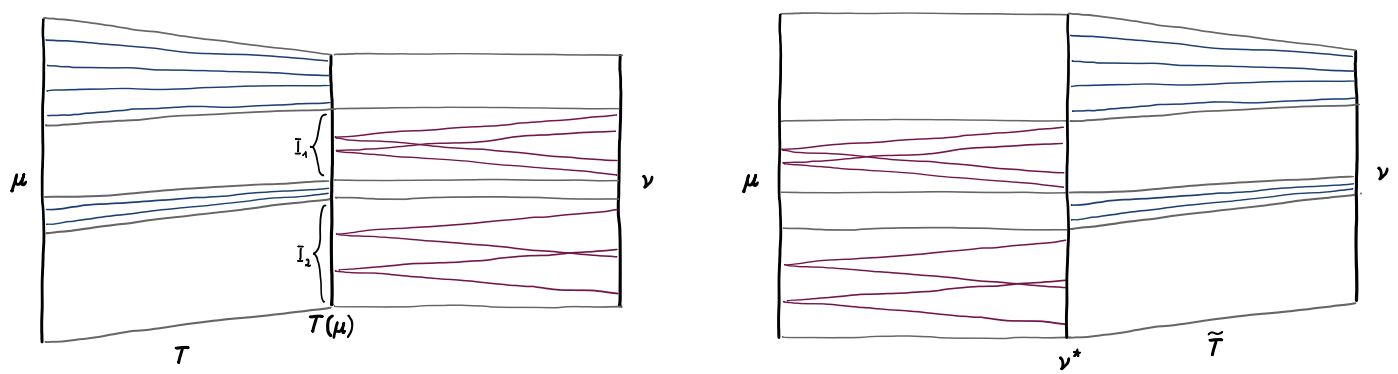

Figure 1: The solutions to (1.2)/(1.4) and (1.7), respectively. Blue lines depict contractive parts of the map, purple lines depict areas with (non trivial) martingale transport.

\subsection{A reverse problem}

Alfonsi, Corbetta, and Jourdain [2] proved that the same value is obtained when reversing the order of transport and convex order relaxation in (1.2), i.e.

$$
V_{\theta}(\mu, \nu)=\inf _{\mu \leq_{c} \nu^{*}} W_{\theta}\left(\nu^{*}, \nu\right) ;
$$

moreover they find ([2, Proposition 4.3]) a monotone mapping which is optimal between the optimizer of (1.7) and $\nu$ as well as between $\mu$ and the optimizer of (1.2).

As a counterpart to Theorems 1.2 and 1.3 we establish the following result, strengthening the connection between (1.2) and (1.7).

Theorem 1.4. Let $\mu, \nu \in \mathcal{P}_{1}(\mathbb{R})$. Then there exists a unique $\leq_{c}$-smallest measure $\nu^{*}$, $\mu \leq_{c} \nu^{*}$, which can be pushed onto $\nu$ by an increasing 1-Lipschitz mapping. Moreover we have

1. If $\theta: \mathbb{R} \rightarrow \mathbb{R}$ is convex then $\nu^{*}$ is an optimizer of (1.7). If $\theta: \mathbb{R} \rightarrow \mathbb{R}$ is strictly convex and $V_{\theta}(\mu, \nu)$ is finite, $\nu^{*}$ is the unique optimizer of (1.7).

2. There exists a ( $\nu^{*}$-unique) increasing 1-Lipschitz mapping $\tilde{T}$ which pushes $\nu^{*}$ onto $\nu ; \tilde{T}$ has slope 1 on each interval $I$, where $I$ is irreducible w.r.t. $\left(\mu, \nu^{*}\right)$.

3. $\tilde{T}$ is the weak monotone rearrangement between $\mu$ and $\nu$.

As in the previous section, (1.7) could be interpreted as a concatenation of a martingale transport with the weak monotone rearrangement. 


\subsection{An auxiliary result}

We close this introductory section with an auxiliary result that will be important in the proofs of our results. Since it might be of independent interest we provide the $d$-dimensional version. We denote the topology induced by the $\rho$-Wasserstein distance on the space of probability measures on $\mathbb{R}^{d}$ by $\mathcal{W}_{\rho}$.

Theorem 1.5 (Stability). Let $1 \leq \rho<\infty,\left(\mu^{k}\right)_{k \in \mathbb{N}} \in \mathcal{P}_{\rho}\left(\mathbb{R}^{d}\right)^{\mathbb{N}},\left(\nu^{k}\right)_{k \in \mathbb{N}} \in \mathcal{P}_{1}\left(\mathbb{R}^{d}\right)^{\mathbb{N}}$ and $\theta: \mathbb{R}^{d} \rightarrow \mathbb{R}$ convex and such that for some constant $c>0$ it holds

$$
\theta(x) \leq c\left(1+|x|^{\rho}\right) \quad \forall x \in \mathbb{R}^{d} .
$$

If $\mu^{k} \rightarrow \mu$ in $\mathcal{W}_{\rho}$ and $\nu^{k} \rightarrow \nu$ in $\mathcal{W}_{1}$, then $\lim _{k} V_{\theta}\left(\mu^{k}, \nu^{k}\right)=V_{\theta}(\mu, \nu)$. If additionally $\theta$ is strictly convex, we have that

1. $\arg \min _{\eta \leq_{c} \nu^{k}} W_{\theta}\left(\mu^{k}, \nu^{k}\right) \rightarrow \arg \min _{\eta \leq_{c} \nu} W_{\theta}(\mu, \nu) \quad$ in $\mathcal{W}_{1}$,

2. the sequence of maps $T^{k}$, where $T^{k}(\mu) \leq_{c} \nu$ and $V_{\theta}\left(\mu, \nu^{k}\right)=W_{\theta}\left(\mu, T^{k}(\mu)\right)$, converges in $\mu$-probability to $T$, where $T(\mu) \leq_{c} \nu$ and $V_{\theta}(\mu, \nu)=W_{\theta}(\mu, T(\mu))$.

\section{C-Monotonicity implies geometric characterization}

In this part we prove the following

Theorem 2.1. Let $\mu, \nu \in \mathcal{P}_{1}(\mathbb{R})$ and $\theta: \mathbb{R} \rightarrow \mathbb{R}$ strictly convex. If $V_{\theta}(\mu, \nu)$ yields a finite value, for any optimizer $\pi \in \Pi(\mu, \nu)$ of (1.4), the map

$$
T(x):=\int_{\mathbb{R}} y \pi_{x}(d y),
$$

is independent of the specific optimizer $\pi$. This map is further admissible in the sense of Definition 1.1 and it has slope 1 on $T^{-1}(I)$ if $I$ is an irreducible interval for $(T(\mu), \nu)$.

To prove Theorem 2.1, we need some further properties connected to irreducibility:

Lemma 2.2. Suppose $\left\{u_{\mu}<u_{\nu}\right\}=:(a, b)$. Then for any $\pi \in \Pi_{M}(\mu, \nu)$, any regular disintegration $\left(\pi_{x}\right)_{x \in \mathbb{R}}$ w.r.t. $\mu$ and any $c \in(a, b)$ such that $\mu((a, c))>0, \mu((c, b))>0$, there are $x \in(a, c], y \in[c, b), x \neq y$, such that the supports of $\pi_{x}$ and $\pi_{y}$ overlap, i.e.

$$
\operatorname{int}\left(\operatorname{co}\left(\operatorname{supp}\left(\pi_{x}\right)\right)\right) \cap \operatorname{co}\left(\operatorname{supp}\left(\pi_{y}\right)\right) \cup \operatorname{int}\left(\operatorname{co}\left(\operatorname{supp}\left(\pi_{y}\right)\right)\right) \cap \operatorname{co}\left(\operatorname{supp}\left(\pi_{x}\right)\right) \neq \emptyset .
$$

Proof. To show this assertion, we assume the opposite. So there exist $c \in\left\{u_{\mu}<u_{\nu}\right\}$, $\pi \in \Pi_{M}(\mu, \nu)$ with fixed disintegration $\left(\pi_{x}\right)_{x \in \mathbb{R}}$ w.r.t. $\mu$ and

$$
\mu((a, c))>0, \quad \mu((c, b))>0,
$$

so that for all $x, y$ with $a<x \leq c \leq y<b, x \neq y$, we have

$$
\operatorname{int}\left(\operatorname{co}\left(\operatorname{supp}\left(\pi_{x}\right)\right)\right) \cap \operatorname{co}\left(\operatorname{supp}\left(\pi_{y}\right)\right) \cup \operatorname{int}\left(\operatorname{co}\left(\operatorname{supp}\left(\pi_{y}\right)\right)\right) \cap \operatorname{co}\left(\operatorname{supp}\left(\pi_{x}\right)\right)=\emptyset .
$$

Since $\pi$ is a martingale coupling, and by (2.2), there exists $d \in(a, b)$ with

$$
\begin{array}{cl}
\operatorname{supp}\left(\pi_{x}\right) \subseteq(-\infty, d] & \text { for } \mu \text {-a.e. } x<c, \\
\operatorname{supp}\left(\pi_{y}\right) \subseteq[d, \infty) & \text { for } \mu \text {-a.e. } y>c .
\end{array}
$$

Write $d_{+}$for the largest and $d_{-}$for the smallest $d$ such that (2.3) holds. Note then that $d_{-}, d_{+} \in(a, b)$. We have either $\operatorname{supp}\left(\pi_{c}\right) \subseteq\left[d_{-}, d_{+}\right]$or $\mu(\{c\})=0$, which in any case implies $\mu\left(\left[c \wedge d_{-}, c \vee d_{+}\right] \backslash\{c\}\right)=0$. Thus, we infer

$$
\begin{aligned}
1 & =\pi\left((-\infty, c) \times\left(-\infty, d_{-}\right) \cup\{c\} \times\left[d_{-}, d_{+}\right] \cup(c, \infty) \times\left(d_{+}, \infty\right)\right) \\
& =\pi\left(\left(-\infty, d_{-}\right) \times\left(-\infty, d_{-}\right] \cup\{c\} \times\left[d_{-}, d_{+}\right] \cup\left(d_{+}, \infty\right) \times\left[d_{+}, \infty\right)\right),
\end{aligned}
$$


and we conclude by contradicting $u_{\mu}\left(d_{-}\right)<u_{\nu}\left(d_{-}\right)$since

$$
\begin{aligned}
\int_{\mathbb{R}}\left|x-d_{-}\right| \mu(d x) & =\int_{\left(a, d_{-}\right)}\left|x-d_{-}\right| \mu(d x)+\int_{\left[d_{-}, b\right)}\left|x-d_{-}\right| \mu(d x) \\
& =\int_{\left(a, d_{-}\right)}\left|y-d_{-}\right| \pi_{x}(d y) \mu(d x)+\int_{\left[d_{-}, b\right)}\left|y-d_{-}\right| \pi_{x}(d y) \mu(d x) \\
& =\int_{\mathbb{R}}\left|x-d_{-}\right| \nu(d x) .
\end{aligned}
$$

Lemma 2.3. Let $p, q \in \mathcal{P}_{1}(\mathbb{R})$ have overlapping supports (cf. (2.1)). Then there exists a continuous map $[0,1] \ni \alpha \mapsto\left(p_{\alpha}, q_{\alpha}\right) \in \mathcal{P}(\mathbb{R}) \times \mathcal{P}(\mathbb{R})$ such that

$$
p_{\alpha}+q_{\alpha}=p+q \quad \forall \alpha \in[0,1]
$$

and such that for some $\beta \in(0,1)$ the functions

$$
[\beta, 1] \ni \alpha \mapsto \int_{\mathbb{R}} z p_{\alpha}(d z), \quad[\beta, 1] \ni \alpha \mapsto \int_{\mathbb{R}} z q_{\alpha}(d z)
$$

are strictly increasing and decreasing, respectively.

Proof. Let $\alpha \in[0,1]$ and define the inverse distribution functions by

$$
s_{\alpha}:=\inf \left\{x \in \mathbb{R}: F_{p}(x) \geq \alpha\right\}, \quad t_{\alpha}:=\inf \left\{x \in \mathbb{R}: F_{q}(x) \geq \alpha\right\},
$$

where $F_{p}$ and $F_{q}$ denote the cumulative distribution functions of $p$ and $q$, respectively. Define two auxiliary measures

$$
\tilde{p}_{\alpha}:=\left.p\right|_{\left(-\infty, s_{\alpha}\right)}+\left(\alpha-F_{p}\left(s_{\alpha}-\right)\right) \delta_{\left\{s_{\alpha}\right\}}, \quad \tilde{q}_{\alpha}:=\left.q\right|_{\left(-\infty, t_{\alpha}\right)}+\left(\alpha-F_{q}\left(t_{\alpha}-\right)\right) \delta_{\left\{t_{\alpha}\right\}} .
$$

We define the probability measure $p_{\alpha}$ by taking the leftmost $\alpha$ mass of $p$ and the leftmost $1-\alpha$ mass of $q$, i.e., let $p_{\alpha}$ be given by $p_{\alpha}:=\tilde{p}_{\alpha}+\tilde{q}_{1-\alpha}$. To satisfy (2.4), we set $q_{\alpha}:=p+q-p_{\alpha}$, which also yields continuity of $\alpha \mapsto\left(p_{\alpha}, q_{\alpha}\right)$. Since the supports of $p$ and $q$ are overlapping, see (2.1), we find constants $c_{1}, c_{2} \in \mathbb{R}$ with $c_{1}>c_{2}$ such that

$$
\alpha_{1}:=p\left(\left[c_{1},+\infty\right)\right)>0 \text { and } \alpha_{2}:=q\left(\left(-\infty, c_{2}\right]\right)>0 .
$$

Let $0<\alpha_{3}<\alpha_{1} \wedge \alpha_{2}$, then for any $1-\alpha_{3} \leq \alpha<\alpha^{\prime} \leq 1$ we have

$$
\begin{aligned}
\int_{\mathbb{R}} z p_{\alpha^{\prime}}(d z)-\int_{\mathbb{R}} z p_{\alpha}(d z) & =\int_{\mathbb{R}} z\left(\tilde{p}_{\alpha^{\prime}}-\tilde{p}_{\alpha}\right)(d z)+\int_{\mathbb{R}} z\left(\tilde{q}_{1-\alpha^{\prime}}-\tilde{q}_{1-\alpha}\right)(d z) \\
& \geq \int_{\mathbb{R}} c_{1}\left(\tilde{p}_{\alpha^{\prime}}-\tilde{p}_{\alpha}\right)(d z)-\int_{\mathbb{R}} c_{2}\left(\tilde{q}_{1-\alpha}-\tilde{q}_{1-\alpha^{\prime}}\right)(d z) \\
& =\left(\alpha^{\prime}-\alpha\right)\left(c_{1}-c_{2}\right)>0
\end{aligned}
$$

and conclude that for $\beta:=1-\alpha_{3}$ the maps defined in (2.5) are strictly monotone.

An important tool in the proof of Theorem 2.1 is $C$-monotonicity, a concept which was introduced for the weak optimal transport problem in [5, 12, 7]. In the current setting it takes the following form:

Definition 2.4 ( $C$-monotonicity). A coupling $\pi \in \Pi(\mu, \nu)$ is $C$-monotone (with cost $C(x, p)$ $\left.=\theta\left(x-\int y p(d y)\right)\right)$ if there exists a measurable set $\Gamma \subseteq \mathbb{R}$ with $\mu(\Gamma)=1$, such that for any finite number of points $x_{1}, \ldots, x_{N}$ in $\Gamma$ and probability measures $m_{1}, \ldots, m_{N} \in \mathcal{P}_{1}(\mathbb{R})$ with $\sum_{i=1}^{N} m_{i}=\sum_{i=1}^{N} \pi_{x_{i}}$, we have

$$
\sum_{i=1}^{N} \theta\left(x_{i}-\int y \pi_{x_{i}}(d y)\right) \leq \sum_{i=1}^{N} \theta\left(x_{i}-\int y m_{i}(d y)\right) .
$$




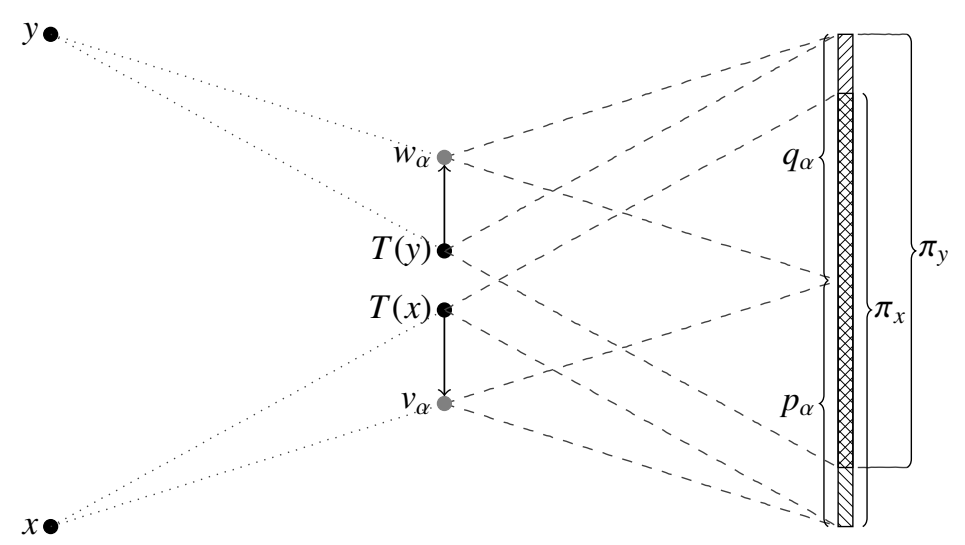

Figure 2: Sketch of usage of Lemma 2.2 and Lemma 2.3 to find contradiction to $C$ monotonicity of an $C$-optimal coupling $\pi$.

Proof of Theorem 2.1. Let $\pi^{*}$ and $\tilde{\pi}^{*}$ be optimal for the weak optimal transport problem (1.4). Clearly, $\frac{\pi^{*}+\tilde{\pi}^{*}}{2}$ constitutes another optimizer of (1.4). Strict convexity of $\theta$ yields that

$$
\int_{\mathbb{R}} y \pi_{x}^{*}(d y)=\int_{\mathbb{R}} y \frac{\pi^{*}+\tilde{\pi}^{*}}{2}(d y)=\int_{\mathbb{R}} y \tilde{\pi}_{x}^{*}(d y) \quad \mu \text {-a.e. } x,
$$

showing that $T$ is independent of the choice of the optimizer.

By the monotonicity principle [7, Theorem 5.2$] \pi^{*}$ is $C$-monotone, therefore, there exists a set $\Gamma \subseteq \mathbb{R}$ with $\mu(\Gamma)=1$ and such that for all $x, y \in \Gamma, p_{1}, p_{2} \in \mathcal{P}_{1}(\mathbb{R})$ satisfying $\pi_{x}^{*}+\pi_{y}^{*}=p_{1}+p_{2}$, we have

$$
\theta\left(x-\int_{\mathbb{R}} z \pi_{x}^{*}(d y)\right)+\theta\left(y-\int_{\mathbb{R}} z \pi_{y}^{*}(d z)\right) \leq \theta\left(x-\int_{\mathbb{R}} z p_{1}(d z)\right)+\theta\left(y-\int_{\mathbb{R}} z p_{2}(d z)\right) .
$$

Let $T(x)=\int_{\mathbb{R}} y \pi_{x}^{*}(d y)$. If $x<y$ and $T(y)<T(x)$ we would have by strict convexity that

$$
\frac{\theta(x-T(y))-\theta(x-T(x))}{x-T(y)-(x-T(x))}<\frac{\theta(y-T(y))-\theta(y-T(x))}{y-T(y)-(y-T(x))},
$$

which then would contradict (2.6) for the choice $p_{1}=\pi_{y}^{*}$ and $p_{2}=\pi_{x}^{*}$. Hence $T$ is $\mu$-almost surely increasing. Letting $x, y \in \Gamma$, for any $\alpha \in(0,1]$ we define the convex combination of $\pi_{x}^{*}$ and $\pi_{y}^{*}$ by

$$
p_{1}^{\alpha}=(1-\alpha) \pi_{x}^{*}+\alpha \pi_{y}^{*}, \quad p_{2}^{\alpha}=\alpha \pi_{x}^{*}+(1-\alpha) \pi_{y}^{*} .
$$

Due to $T$ being a monotone map, we can assume that $x \leq y$ and $T(x) \leq T(y)$. Plugging $p_{1}^{\alpha}$ and $p_{2}^{\alpha}$ into (2.6), dividing this inequality by $\alpha$, and sending $\alpha \searrow 0$, yields

$$
\left(\partial_{+} \theta(x-T(x))-\partial_{-} \theta(y-T(y))\right)(T(x)-T(y)) \geq 0,
$$

where $\partial_{+} \theta$ denotes the right-hand side derivative and $\partial_{-} \theta$ the left-hand side derivative, respectively. By convexity of $\theta$, (2.7) is equivalent to

$$
(x-T(x)-y+T(y))(T(x)-T(y)) \geq 0 .
$$

Hence, $|x-y| \geq|T(x)-T(y)|$ and $T$ is $\mu$-a.e. 1-Lipschitz.

Further, we have that $T(\mu) \leq_{c} \nu$ and $\bar{\pi}:=(T, i d)\left(\pi^{*}\right) \in \Pi_{M}(T(\mu), \nu)$. Since the cost only depends on $x$ and $T(x)$, but not on the martingale part (i.e., the part which 
transports mass from $T(x)$ according to $\pi_{x}^{*}(d y)$ ), it could happen that $T$ is constant on some set $I$, i.e., $\forall x, y \in I$ we have

$$
\int_{\mathbb{R}} z \pi_{x}^{*}(d z)=\int_{\mathbb{R}} z \pi_{y}^{*}(d z),
$$

but $\pi_{x}^{*}$ and $\pi_{y}^{*}$ do not have to match necessarily. Hence, by choosing some other optimizer (the one which averages on $I$ over all $\pi_{z}^{*}$ appropriately), we may assume that $\pi_{x}^{*}$ is constant on $I$. Therefore, we assume without loss of generality $\bar{\pi}_{T(x)}=\pi_{x}^{*}$, $\mu$-a.e. Let $\left(I_{k}\right)_{k \in \mathbb{N}}$ be the intervals given by the decomposition of $(T(\mu), \nu)$ into irreducible intervals. Assume that there is an interval $I_{k}$ so that on $T^{-1}\left(I_{k}\right)$ the map $T$ does not have $\mu$-a.s. slope 1. Then Lemma 2.2 provides two points $\tilde{x}, \tilde{y}$ in $T\left(\Gamma \cap I_{k}\right)$ and two corresponding points $x, y \in \Gamma \cap I_{k}$ such that

$$
x<y, \quad T(x)=\tilde{x}<\tilde{y}=T(y), \quad x-T(x)>y-T(y),
$$

and the overlapping condition (2.1) holds for $\bar{\pi}_{T(x)}=\pi_{x}^{*}, \bar{\pi}_{T(y)}=\pi_{y}^{*}$. Lemma 2.3 allows us to define measures $p_{\alpha}$ and $q_{\alpha}$ on $\mathbb{R}$ such that

$$
\pi_{x}^{*}+\pi_{y}^{*}=\bar{\pi}_{T(x)}+\bar{\pi}_{T(y)}=p_{\alpha}+q_{\alpha} .
$$

For a graphical depiction compare with Figure 2. Hence,

$$
T(x)>v_{\alpha}:=\int_{\mathbb{R}} z p_{\alpha}(d z), \quad T(y)<w_{\alpha}:=\int_{\mathbb{R}} z q_{\alpha}(d z),
$$

are strictly monotone, continuous maps on $[\beta, 1]$. Therefore, we find $\alpha \in(\beta, 1)$ with

$$
x-v_{1}=x-T(x)<x-v_{\alpha} \leq y-w_{\alpha}<y-T(y)=y-w_{1} .
$$

By strict convexity of $\theta$ we find

$$
\frac{\theta\left(y-w_{\alpha}\right)-\theta(x-T(x))}{y-w_{\alpha}-x+T(x)}<\frac{\theta(y-T(y))-\theta\left(x-\nu_{\alpha}\right)}{y-T(y)-x+\nu_{\alpha}},
$$

which then yields a contradiction to $C$-monotonicity:

$$
\begin{aligned}
\theta\left(x-\int_{\mathbb{R}} z \pi_{x}^{*}(d z)\right)+\theta\left(y-\int_{\mathbb{R}} z \pi_{y}^{*}(d z)\right) & =\theta(x-T(x))+\theta(y-T(y)) \\
& >\theta\left(x-v_{\alpha}\right)+\theta\left(y-w_{\alpha}\right) \\
& =\theta\left(x-\int_{\mathbb{R}} z p_{\alpha}(d z)\right)+\theta\left(y-\int_{\mathbb{R}} z q_{\alpha}(d z)\right) .
\end{aligned}
$$

\section{Sufficiency of the geometric characterization}

Naturally the question arises whether any map $T$ satisfying the properties in Theorem 2.1 must be optimal. The aim of this section is to establish this:

Theorem 3.1. Let $\mu, \nu \in \mathcal{P}_{1}(\mathbb{R})$. Then any coupling $\pi \in \Pi(\mu, \nu)$ for which $T(x):=$ $\int_{\mathbb{R}} y \pi_{x}(d y)$ is admissible (in the sense of Definition 1.1) with slope 1 on each interval $T^{-1}(I)$, where $I$ is irreducible w.r.t. $(T(\mu), \nu)$, is optimal for (1.4), i.e.,

$$
V_{\theta}(\mu, \nu)=\int_{\mathbb{R}} \theta(x-T(x)) \mu(d x) .
$$

The proof is based on dual optimizers and their explicit representation. As long as $T$ is strictly increasing, [19, Theorem 2.1] provides dual optimizers to $V_{\theta}(\mu, T(\mu))$. Investigating dual optimizers further, we are able to show here $V_{\theta}(\mu, T(\mu))=V_{\theta}(\mu, \nu)$. First, Lemma 3.2 helps us to carefully approximate the increasing map $T$ with strictly increasing maps $T_{\varepsilon}$. 
Lemma 3.2. Let $T: \mathbb{R} \rightarrow \mathbb{R}$ be an increasing map, with $T-i d$ decreasing. Then, for any $\varepsilon>0$ there is a strictly increasing map $T_{\varepsilon}: \mathbb{R} \rightarrow \mathbb{R}$, with $T_{\varepsilon}-i d$ decreasing, such that

$$
\left|T-T_{\varepsilon}\right|_{\infty} \leq \varepsilon,
$$

and $T$ is affine with slope 1 on an interval $I$ if and only if $T_{\varepsilon}$ is affine with slope 1 on $I$.

Proof. Since $T$ is increasing we know that the pre-image of any point under $T$ corresponds to an interval. Therefore, we can find at most countable many, disjoint intervals $\left(I_{k}\right)_{k \in \mathbb{N}}$ of finite length, where $T\left(I_{k}\right)$ is a singleton and $T$ is strictly increasing on the complement, i.e., on $\bigcap_{k} I_{k}^{c}$. For any $\varepsilon>0$, we define $g_{\varepsilon}: \mathbb{R} \rightarrow \mathbb{R}$

$$
g_{\varepsilon}(x):= \begin{cases}\frac{\varepsilon}{\lambda\left(I_{k}\right) 2^{k}} & \exists k \in \mathbb{N}: x \in I_{k}, \\ 0 & \text { else. }\end{cases}
$$

Then the map $T_{\varepsilon}(x):=T(x)+\int_{-\infty}^{x}\left[g_{\varepsilon}(y) \wedge 1\right] d y$ satisfies the desired properties.

Let $T: \mathbb{R} \rightarrow \mathbb{R}$ be an increasing 1-Lipschitz function. Then $T$ induces a unique decomposition of $\mathbb{R}$ into at most countably many maximal, closed, disjoint intervals $\left(I_{k}\right)_{k}$ and a (G $G_{\delta}$-set) $G$ such that for all $k \in \mathbb{N}$ the map $\left.T\right|_{I_{k}}$ is affine with slope 1 and $\left.T\right|_{G}$ is properly contractive, i.e., for any two points $x, y \in G$ we have $|T(x)-T(y)|<|x-y|$. Below we call the intervals $\left(I_{k}\right)_{k}$ irreducible w.r.t. $T$.

Proof of Theorem 3.1. The convex function $\theta$ can be approximated by an increasing sequence of convex Lipschitz functions $\left(\theta_{n}\right)_{n \in \mathbb{N}}$, e.g.

$\theta_{n}(x):=\theta(x) 1_{[-n, n]}(x)+\left[\theta(n)+(x-n) \partial_{+} \theta(n)\right] 1_{(n,+\infty)}+\left[\theta(-n)+(x+n) \partial_{-} \theta(-n)\right] 1_{(-\infty,-n)}$.

By monotone convergence we find ${ }^{1} \sup _{n} V_{\theta_{n}}(\mu, \nu)=V_{\theta}(\mu, \nu)$. Indeed, if $\pi^{n}$ optimizes $V_{\theta_{n}}(\mu, \nu)$ and assuming w.l.o.g. that $\pi_{n} \rightarrow \pi$, then

$$
\begin{aligned}
\lim _{n} \int \theta_{n}\left(x-\int y \pi_{x}^{n}(d y)\right) \mu(d x) & \geq \lim _{n} \int \theta_{m}\left(x-\int y \pi_{x}^{n}(d y)\right) \mu(d x) \\
& \geq \int \theta_{m}\left(x-\int y \pi_{x}(d y)\right) \mu(d x),
\end{aligned}
$$

by [7, Proposition 2.8]. Thus the claim follows by taking the supremum in $m$.

From this, it suffices to consider the case when $\theta$ is Lipschitz continuous. By Lemma 3.2 we find for any $\varepsilon>0$ a strictly increasing map $T_{\varepsilon}$, such that $T_{\varepsilon}-i d$ is decreasing, the decompositions of $T$ and $T_{\varepsilon}$ match (i.e., $T$ is affine with slope 1 on an interval $I$ iff $T_{\varepsilon}$ is affine with slope 1 on $I$ ), and $\left|T_{\varepsilon}-T\right|_{\infty} \leq \varepsilon$. Then [19, Theorem 2.1] provides a convex, Lipschitz continuous function $f_{\varepsilon}: \mathbb{R} \rightarrow \mathbb{R}$ such that for all $x \in \mathbb{R}$

$$
R f_{\varepsilon}(x):=\inf _{y \in \mathbb{R}} f_{\varepsilon}(y)+\theta(x-y)=f_{\varepsilon}\left(T_{\varepsilon}(x)\right)+\theta\left(x-T_{\varepsilon}(x)\right),
$$

which is even affine on the parts where $T_{\varepsilon}$ is affine. Write $S_{\varepsilon}(x)=\int_{-\infty}^{x}\left[g_{\varepsilon}(z) \wedge 1\right] d z$, where $g_{\varepsilon}$ is defined as in (3.1) so $T_{\varepsilon}=T+S_{\varepsilon}$. In the following we will show that $f_{\varepsilon}$ is a dual optimizer (cf. the dual problem as in [14, Theorem 2.11]) to the weak transport problem $V_{\theta}$ between the marginals of the coupling $\pi^{\varepsilon}$, where $\pi^{\varepsilon}$ is defined as the push-forward measure of $\pi$ by the function

$$
(x, y) \mapsto\left(x, y+S_{\varepsilon}(x)\right) .
$$

First, we compute the barycenters of $\pi_{x}^{\varepsilon}$ :

$$
\int_{\mathbb{R}} y \pi_{x}^{\varepsilon}(d y)=\int_{\mathbb{R}} y+S_{\varepsilon}(x) \pi_{x}(d y)=T_{\varepsilon}(x),
$$

\footnotetext{
${ }^{1}$ Recall that by [13] the optimizer of the weak transport problem does not depend on the convex cost.
} 
and $T_{\varepsilon}(\mu) \leq_{c} \operatorname{proj}_{2}\left(\pi^{\varepsilon}\right)=: \nu_{\varepsilon}$. Given the sets $\left(I_{k}\right)_{k \in \mathbb{N}}$ and $F$ from the decomposition of $(T(\mu), \nu)$ into irreducible intervals, we find the sets $\left(I_{k}^{\varepsilon}\right)_{k \in \mathbb{N}}$ and $F_{\varepsilon}$ from the decomposition of $\left(T_{\varepsilon}(\mu), \nu_{\varepsilon}\right)$ into irreducible intervals by setting

$$
I_{k}^{\varepsilon}:=I_{k}+S_{\varepsilon}(x) \text { for any } y \in I_{k} \text { and } x \in T^{-1}(y), \quad F_{\varepsilon}:=\bigcap_{k}\left(I_{k}^{\varepsilon}\right)^{c} .
$$

In view of the structure of martingale couplings, see [8, Theorem A.4], we find that for $\mu$-a.e. $x \in T_{\varepsilon}^{-1}\left(I_{k}^{\varepsilon}\right)$ we have $\operatorname{supp}\left(\pi_{x}^{\varepsilon}\right) \subseteq \bar{I}_{k}^{\varepsilon}$ and $\pi_{x}^{\varepsilon}=\delta_{T(x)+S_{\varepsilon}(x)} \mu$-a.e. on $F_{\varepsilon}$. Since $T$ has slope 1 on each interval $I$ which is irreducible w.r.t. $(T(\mu), \nu)$, we infer the same for the map $T_{\varepsilon}$ and the irreducible intervals of $\left(T_{\varepsilon}(\mu), \nu_{\varepsilon}\right)$. The next computation establishes duality of the pair $\left(\pi^{\varepsilon}, f_{\varepsilon}\right)$, where we use affinity of $f_{\varepsilon}$ on the irreducible components of $\left(T_{\varepsilon}(\mu), \nu_{\varepsilon}\right)$ :

$$
\begin{aligned}
\int_{\mathbb{R}} \theta\left(x-\int_{\mathbb{R}} y \pi_{x}^{\varepsilon}(d y)\right) \mu(d x) & =\int_{\mathbb{R}} \theta\left(x-T_{\varepsilon}(x)\right) \mu(d x)=\int_{\mathbb{R}} R f_{\varepsilon}(x)-f_{\varepsilon}\left(T_{\varepsilon}(x)\right) \mu(d x) \\
& =\int_{\mathbb{R}} R f_{\varepsilon}(x) \mu(d x)-\int_{\mathbb{R}} f_{\varepsilon}\left(\int_{\mathbb{R}} y \pi_{x}^{\varepsilon}(d y)\right) \mu(d x) \\
& =\int_{\mathbb{R}} R f_{\varepsilon}(x) \mu(d x)-\int_{\mathbb{R}} f_{\varepsilon}(y) \nu_{\varepsilon}(d y) .
\end{aligned}
$$

This easily proves that $\pi^{\varepsilon}$ is optimal for the optimal weak transport problem $V_{\theta}\left(\mu, \nu_{\varepsilon}\right)$. Drawing the limit for $\varepsilon \searrow 0$, we observe

$$
V_{\theta}\left(\mu, \nu_{\varepsilon}\right)=\int_{\mathbb{R}} \theta\left(x-T_{\varepsilon}(x)\right) \mu(d x) \rightarrow \int_{\mathbb{R}} \theta(x-T(x)) \mu(d x) .
$$

As $\theta$ is Lipschitz, we can apply stability Theorem 1.5 and obtain optimality of $\pi$.

\section{Geometry of the weak monotone rearrangement}

We can summarize Theorems 2.1 and 3.1 as follows: There exists an admissible map $T$ with slope 1 on $T^{-1}(I)$ whenever $I$ is an irreducible interval w.r.t. $(T(\mu), \nu)$, such that $\pi$ is optimal for (1.4) iff $T(x)=\int y \pi_{x}(d y)$ ( $\mu$-a.s.). We now show that this map is the weak monotone rearrangement and is therefore the maximum in convex order of the set ${ }^{2}$

$$
M(\mu, \nu):=\left\{S: \mathbb{R} \rightarrow \mathbb{R}: S \text { is increasing and 1-Lipschitz, } S(\mu) \leq_{c} \nu\right\} .
$$

Heuristically speaking, if the maximum in convex order of the set $M(\mu, \nu)$ is again given by an increasing, 1-Lipschitz map, then this map is as close as possible to a shifted identity. In turn, this is favourable when trying to find the minimum in convex order of

$$
\{(i d-S)(\mu): S \in M(\mu, \nu)\},
$$

which gives reason to why there should exist a single optimizer to (1.4) for all convex $\theta$.

As preparation to establishing Theorem 1.3, we prove Lemma 4.1 and Lemma 4.2.

Lemma 4.1. Let $\mu \in \mathcal{P}_{1}(\mathbb{R}), T, S: \mathbb{R} \rightarrow \mathbb{R}$ be increasing maps with

$$
\int_{\mathbb{R}} T(x) \mu(d x)=\int_{\mathbb{R}} S(x) \mu(d x),
$$

then the maximum w.r.t. the convex order ${ }^{3}$ of $T(\mu)$ and $S(\mu)$ is given by $T^{*}(\mu)$ for $T^{*}$ an increasing map. If in addition $T, S$ are L-Lipschitz with $L>0$, then $T^{*}$ is also L-Lipschitz.

\footnotetext{
${ }^{2}$ We abuse terminology here, meaning maximum of $\{S(\mu): S \in M(\mu, \nu)\}$.

${ }^{3}$ The maximum in convex order of $\nu, \eta \in \mathcal{P}_{1}(\mathbb{R})$, denoted $\nu \vee \eta$, satisfies $\nu, \eta \leq_{c} \nu \vee \eta$ as well as $\nu \vee \eta \leq_{c} \xi$ whenever $\nu, \eta \leq_{c} \xi$. One can easily characterize it by the identity $u_{\nu \vee \eta}(\cdot)=u_{\nu}(\cdot) \vee u_{\eta}(\cdot)$. Similarly, the minimum of $\nu$ and $\eta$, denoted $\nu \wedge \eta$, is characterized by $u_{\nu \wedge \eta}$ being equal to the greatest convex minorant of $u_{\nu} \wedge u_{\eta}$.
} 
Proof. The maximum of $T(\mu)$ and $S(\mu)$ w.r.t. the convex order is uniquely determined by the maximum of its potential functions, i.e. $u_{S(\mu)} \vee u_{T(\mu)}=u_{S(\mu) \vee T(\mu)}$. The right-hand side derivative of the potential function can be expressed by the cumulative distribution function, namely $\partial_{+} u_{\mu}(x)=2 F_{\mu}(x)-1$. By continuity of the potential functions, we find a minimal partition of $\mathbb{R}$ into at most countably many disjoint intervals $\left(\left(l_{k}, r_{k}\right]\right)_{k \in \mathbb{N}}$, where $u_{T(\mu)}=u_{S(\mu)}$ on $\left\{l_{k}, r_{k}\right\}$, and restricted onto $\left(l_{k}, r_{k}\right]$ one of the following holds true:

(a) $\left.u_{S(\mu)}\right|_{\left(l_{k}, r_{k}\right]} \leq\left. u_{T(\mu)}\right|_{\left(l_{k}, r_{k}\right]}$,

(b) $\left.u_{S(\mu)}\right|_{\left(l_{k}, r_{k}\right]} \geq\left. u_{T(\mu)}\right|_{\left(l_{k}, r_{k}\right]}$.

Suppose w.l.o.g. (a) holds, then

$$
F_{S(\mu) \vee T(\mu)}(x)=F_{T(\mu)}(x) \quad x \in\left(l_{k}, r_{k}\right] .
$$

By monotonicity of the maps $S$ and $T$, we can define $T^{*}$ on $\left[\tilde{l}_{k}, \tilde{r}_{k}\right)$ where ${ }^{4}$

$$
\tilde{l}_{k}=\sup T^{-1}\left(\left\{l_{k}\right\}\right) \vee \sup S^{-1}\left(\left\{l_{k}\right\}\right), \quad \tilde{r}_{k}=\sup T^{-1}\left(\left\{r_{k}\right\}\right) \vee \sup S^{-1}\left(\left\{r_{k}\right\}\right),
$$

by

$$
T^{*}(x)= \begin{cases}T(x) & x \in T^{-1}\left(\left(l_{k}, r_{k}\right]\right) \cap\left(\tilde{l}_{k}, \tilde{r}_{k}\right], \\ r_{k} & x \in\left(\tilde{l}_{k}, \tilde{r}_{k}\right] \backslash T^{-1}\left(\left(l_{k}, r_{k}\right]\right) .\end{cases}
$$

Hence, $T^{*}$ is an increasing map and

$$
\begin{gathered}
F_{T^{*}(\mu)}(x)=F_{T(\mu)}(x)=F_{T(\mu) \vee S(\mu)}(x) \quad x \in\left(l_{k}, r_{k}\right), \\
F_{T^{*}(\mu)}\left(r_{k}\right)=F_{S(\mu)}\left(r_{k}\right)=F_{T(\mu) \vee S(\mu)}\left(r_{k}\right) .
\end{gathered}
$$

Therefore, $F_{T^{*}(\mu)}=F_{S(\mu) \vee T(\mu)}$ which is equivalent to $S(\mu) \vee T(\mu)$ being given by the map $T^{*}$. If $T$ and $S$ are in addition $L$-Lipschitz, it follows by construction that $T^{*}$ is $L$-Lipschitz.

Lemma 4.2. Let $\eta_{1} \leq_{c} \eta_{2}$ and $T_{2}\left(\eta_{2}\right) \leq_{c} T_{1}\left(\eta_{1}\right)$, where $T_{1}, T_{2}$ are increasing and 1Lipschitz. Then $\left(i d-T_{1}\right)\left(\eta_{1}\right) \leq_{c}\left(i d-T_{2}\right)\left(\eta_{2}\right)$.

In particular, if $T$ and $S$ are increasing 1-Lipschitz maps s.t. $\int T(x) \mu(d x)=\int S(x) \mu(d x)$, and we denote by $R$ the increasing 1-Lipschitz map with $R(\mu)=S(\mu) \vee T(\mu)$, which exists by Lemma 4.1, then $(i d-R)(\mu) \leq_{c}(i d-S)(\mu) \wedge(i d-T)(\mu)$.

Proof. By [18, Theorem 3.A.5] we have that for any two probability measures $\mu$ and $\nu$ on $\mathbb{R}$ the convex order, i.e., $\mu \leq_{c} \nu$, is characterized by

$$
\int_{0}^{\alpha} F_{\mu}^{-1}(u) d u \geq \int_{0}^{\alpha} F_{\nu}^{-1}(u) d u \quad \forall \alpha \in[0,1],
$$

with equality when $\alpha=1$. For any increasing, continuous map $U: \mathbb{R} \rightarrow \mathbb{R}$, any measure $\mu \in \mathcal{P}(\mathbb{R})$ and $u \in(0,1)$ we find

$$
\begin{aligned}
F_{U(\mu)}^{-1}(u) & =\inf \left\{x \in \mathbb{R}: F_{U(\mu)}(x) \geq u\right\} \\
& =\inf \left\{x \in \mathbb{R}: F_{\mu}\left(\sup U^{-1}(\{x\})\right) \geq u\right\} \\
& =\inf \left\{U(y): y \in \mathbb{R} \text { and } F_{\mu}(y) \geq u\right\} \\
& =U\left(F_{\mu}^{-1}(u)\right) .
\end{aligned}
$$

\footnotetext{
${ }^{4}$ We use the convention that the maximum/supremum of the empty set equals $-\infty$ and the preimage under $T$ and $S$ of $\pm \infty$ is $\pm \infty$.
} 
Now all tools are assembled to show the assertion. Note that $\left(i d-T_{i}\right), i=1,2$, are increasing maps and using the equations (4.3) and (4.4), we compute for $\alpha \in[0,1]$

$$
\begin{aligned}
\int_{0}^{\alpha} F_{\left(i d-T_{1}\right)\left(\eta_{1}\right)}^{-1}(u) d u & =\int_{0}^{\alpha}\left(i d-T_{1}\right)\left(F_{\eta_{1}}^{-1}(u)\right) d u=\int_{0}^{\alpha} F_{\eta_{1}}(u)-F_{T_{1}\left(\eta_{1}\right)}^{-1}(u) d u \\
& \geq \int_{0}^{\alpha} F_{\eta_{2}}(u)-F_{T_{2}\left(\eta_{2}\right)}^{-1}(u) d u=\int_{0}^{\alpha} F_{\left(i d-T_{2}\right)\left(\eta_{2}\right)}^{-1}(u) d u .
\end{aligned}
$$

Proof of Theorem 1.3. Existence of an admissible map $T$ which has slope 1 on each interval $T^{-1}(I)$, where $I$ is irreducible w.r.t. $(T(\mu), \nu)$, was already shown in Theorem 2.1. Therefore, it remains to show that the map is maximal. Denote by $T$ the map given by Theorem 2.1 associated with an optimizer to (1.4) and some strictly convex $\theta: \mathbb{R} \rightarrow \mathbb{R}$. Let $S$ be an arbitrary map in $M(\mu, \nu)$. Then Lemma 4.2 states that

$$
(i d-R)(\mu) \leq_{c}(i d-T)(\mu),
$$

where $R$ is defined as the increasing, 1-Lipschitz map such that $R(\mu)=S(\mu) \vee T(\mu)$. Additionally to existence, strict convexity of $\theta$ ensures $\mu$-almost sure uniqueness of $T$ in the sense that for any optimal coupling $\pi$ we have $\int_{\mathbb{R}} y \pi_{x}(d y)=T(x) \quad \mu$-a.s. Thus, $R(\mu)=T(\mu)$ and $T=R \mu$-almost surely.

Proof of Theorem 1.2. This is a direct consequence of Theorem 2.1, which provides existence of a map with the desired geometric properties, and Theorem 1.3, which provides the equivalence between the geometric properties and maximality.

\section{On the reverse problem of Alfonsi, Corbetta, and Jourdain}

We aim to prove Theorem 1.4 pertaining the reverse problem (1.7).

Lemma 5.1. Let $\eta_{1}, \eta_{2} \in \mathcal{P}_{1}(\mathbb{R})$ with $\int_{\mathbb{R}} y \eta_{1}(d y)=\int_{\mathbb{R}} y \eta_{2}(d y)$, and $T_{1}, T_{2}: \mathbb{R} \rightarrow \mathbb{R}$ be increasing maps with

$$
T_{1}\left(\eta_{1}\right)=T_{2}\left(\eta_{2}\right)=: \nu .
$$

Denote the minimum in convex order of $\eta_{1}$ and $\eta_{2}$ by $\eta$. Then there exists an increasing map $T^{*}$ such that $T^{*}(\eta)=\nu$. If in addition, the maps are $L$-Lipschitz with $L>0$, then the same holds true for $T^{*}$.

Proof. Suppose there exist increasing maps $T_{i}$ and measures $\eta_{i}, i=1,2$, such that $T_{1}\left(\eta_{1}\right)=\nu=T\left(\eta_{2}\right)$. Then the potential function of the minimum $\eta$ of $\eta_{1}$ and $\eta_{2}$ w.r.t. the convex order is given by the convex hull of $u_{\eta_{1}}$ and $u_{\eta_{2}}$. The potential function $u_{\eta}$ completely specifies the cumulative distribution function through $\partial_{+} u_{\eta}=2 F_{\eta}-1$. Thus, we can find a partition of $\mathbb{R}$ into countably many, disjoint intervals $I_{k}=\left[a_{k}, b_{k}\right) \cap \mathbb{R}$. For $k \in \mathbb{N}$ we find $i \neq j \in\{1,2\}$ with $u_{\eta}\left(a_{k}\right)=u_{\eta_{i}}\left(a_{k}\right), u_{\eta}\left(b_{k}\right)=u_{\eta_{j}}\left(b_{k}\right)$ such that one of the following holds

(a) $u_{\eta}(x)=u_{\eta_{i}}(x)$ on $I_{k}$,

(b) $F_{\eta}\left(a_{k}\right)=F_{\eta}\left(b_{k}-\right)=F_{\eta_{j}}\left(b_{k}-\right)$ and $u_{\eta}(x)<u_{\eta_{1}}(x) \wedge u_{\eta_{2}}(x)$ on $\left(a_{k}, b_{k}\right)$.

According to this decomposition, we can define an increasing map $T^{*}$ via

$$
T^{*}(x)= \begin{cases}T_{i}(x) & x \in I_{k},(\mathrm{a}) \text { holds, } \\ T_{i}\left(a_{k}\right) & x \in I_{k}, \text { (b) holds. }\end{cases}
$$

Note that $T^{*}$ is $L$-Lipschitz if $T_{i}, i=1,2$, are $L$-Lipschitz. Let $y \in \mathbb{R}$, due to the continuity of the maps $T_{1}$ and $T_{2}$, we can find points $x_{1}, x_{2} \in \mathbb{R}$ with $p=F_{\nu}(y), x_{1}=F_{\eta_{1}}^{-1}(p) x_{2}=$ 
$F_{\eta_{2}}^{-1}(p)$. Assume that $i=1, j=2$ with $x_{1} \in I_{k}$. If (a) holds, then we have $F_{\eta}(x)=F_{\eta_{1}}(x)$ for all $x \in I_{k}$. Now presume that (b) holds, then $F_{\eta_{2}}\left(b_{k}-\right)=F_{\eta}\left(a_{k}\right) \leq F_{\eta_{1}}\left(x_{1}\right)$. Then

$$
\begin{aligned}
& F_{\eta_{2}}\left(b_{k}\right)=p \Longrightarrow x_{2}=b_{k} \text { and } F_{\eta}\left(b_{k}\right)=p \\
& F_{\eta_{2}}\left(b_{k}\right)<p \Longrightarrow \exists l \in \mathbb{N}: x_{2} \in I_{l} \text { s.t. } i=2 \text { and (a) holds. }
\end{aligned}
$$

Hence, by monotonicity of the map $T^{*}$ we conclude $F_{T^{*}(\eta)}=F_{\nu}$.

Proof of Theorem 1.4. Define $\nu^{*}$ via the weak monotone rearrangement $T$ between $\mu$ and $\nu$, as $\mu$ on the contraction parts of $T$ and accordingly shifted $\nu$ on the affine (irreducible) intervals such that $\tilde{T}\left(\nu^{*}\right)=\nu$. Let $\eta \geq_{c} \mu$. Then for any strictly convex $\theta: \mathbb{R} \rightarrow \mathbb{R}$ and coupling $\pi^{2} \in \Pi(\eta, \nu)$ we have $\int_{\mathbb{R} \times \mathbb{R}} \theta(y-z) \pi^{2}(d y, d z) \geq \int_{\mathbb{R}} \theta\left(y-\int_{\mathbb{R}} z \pi_{y}^{2}(d z)\right) \eta(d y)$, with equality iff $\pi^{2}$ is actually given by a map. Hence, if the optimizer $\pi^{2}$ of $W_{\theta}(\eta, \nu)$ is not given by a map and $\pi^{1} \in \Pi_{M}(\mu, \eta)$, we have

$$
W_{\theta}(\eta, \nu)>\int_{\mathbb{R}} \theta\left(x-\int_{\mathbb{R}} \int_{\mathbb{R}} z \pi_{y}^{2}(d z) \pi_{x}^{1}(d y)\right) \mu(d x) \geq V_{\theta}(\mu, \nu) .
$$

Thus, by the structure of the weak monotone rearrangement, we deduce optimality of $\nu^{*}$ for Problem (1.7). To show uniqueness of (1.7), assume that $\eta$ attains the minimum of (1.7) and the optimizer of $W_{\theta}(\eta, \nu)$ is given by the map $R$. For any martingale coupling $\pi^{1} \in \Pi_{M}(\mu, \eta)$ we define a map by

$$
L(x):=\int_{\mathbb{R}} R(y) \pi_{x}^{1}(d y) .
$$

Then by optimality $L(\mu)=T(\mu)$ and, in particular,

$$
\int_{\mathbb{R}} \theta(y-R(y)) \eta(d y)=\int_{\mathbb{R}} \theta(x-L(x)) \mu(d x)=\int_{\mathbb{R}} \theta(x-T(x)) \mu(d x),
$$

which shows $L=T \mu$-almost surely. By strict convexity, we have

$$
y-R(y)=x-L(x)=x-T(x) \quad \pi^{1} \text {-a.s. }
$$

Since $\pi^{1}$ was arbitrary in $\Pi_{M}(\mu, \eta)$ we get that $R$ is affine with slope 1 on $I$, whenever $I$ is an irreducible interval w.r.t. $(\mu, \eta)$. Therefore $\eta$ and $\nu^{*}$ restricted to $I$ coincide. Hence, $\eta=\nu^{*}$.

We finally show that $\nu^{*}$ is minimal in the convex order as stated. By Lemma 5.1, we can assume $\mu \leq_{c} \eta \leq_{c} \nu^{*}$ and that $\eta$ can be pushed forward onto $\nu$ via an increasing 1-Lipschitz map $S$. It follows by Lemma 4.2 that $(i d-S)(\eta) \leq_{c}(i d-\tilde{T})\left(\nu^{*}\right)$, so

$$
V_{\theta}(\mu, \nu) \leq \int \theta(x-S(x)) \eta(d x) \leq \int \theta(x-\tilde{T}(x)) \nu^{*}(d x)=V_{\theta}(\mu, \nu),
$$

and by the uniqueness obtained above we deduce $\eta=\nu^{*}$.

\section{Stability of barycentric weak transport problems in multiple dimensions}

The final part of the article is concerned with stability of the weak optimal transport problem under barycentric costs, see (1.4). Unlike in the rest of the article we work here on $\mathbb{R}^{d}$. The final aim is to prove Theorem 1.5.

One surprising aspect of this result is that we only require $\nu^{k} \rightarrow \nu$ in $\mathcal{W}_{1}$ and not necessarily in $\mathcal{W}_{\rho}$. This relates to the conditional expectation in (1.4) being 'inside of $\theta$.' We first prove an illuminating intermediate result:

Proposition 6.1. Let $1 \leq \rho<\infty,\left(\mu^{k}\right)_{k \in \mathbb{N}} \in \mathcal{P}_{\rho}\left(\mathbb{R}^{d}\right)^{\mathbb{N}},\left(\nu^{k}\right)_{k \in \mathbb{N}} \in \mathcal{P}_{\rho}\left(\mathbb{R}^{d}\right)^{\mathbb{N}}$ and $\theta: \mathbb{R}^{d} \rightarrow \mathbb{R}$ convex and satisfying the growth condition (1.8). Suppose that $\mu^{k} \rightarrow \mu$ and $\nu^{k} \rightarrow \nu$ in $\mathcal{W}_{\rho}$, and that $\eta \leq_{c} \nu$. Then there exist $\eta^{k} \leq_{c} \nu^{k}$ such that 
(i) $\eta^{k} \rightarrow \eta$ in $\mathcal{W}_{\rho}$,

(ii) $\lim _{k} W_{\theta}\left(\mu^{k}, \eta^{k}\right)=W_{\theta}(\mu, \eta)$.

Proof. It is well-known that $(i)$ together with the stated convergence of the $\mu^{k}$ 's implies (ii), so we proceed to prove the former. Let $\pi^{k}$ be an optimal coupling attaining $W_{\rho}\left(\nu, \nu^{k}\right)$. Let $M$ be any martingale coupling with first marginal $\eta$ and second marginal $\nu$, the existence of which is guaranteed by the assumption $\eta \leq_{c} \nu$ together with Strassen's theorem. We convene on the notation $M(d x, d y)$ and $\pi(d y, d z)$, and define the measure

$$
P(d x, d y, d z)=M_{x}(d x) \pi_{y}^{k}(d z) \nu(d y) .
$$

This measure has $\eta, \nu$ and $\nu^{k}$ as first, second and third marginals. We next define $R^{k}(x)$ as the conditional expectation under $P$ of the third variable given the first one, namely

$$
R^{k}(x)=\iint z \pi_{y}^{k}(d z) M_{x}(d y) .
$$

Next we introduce $\eta^{k}:=R^{k}(\eta)$ so by definition $\eta^{k} \leq_{c} \nu^{k}$. Finally

$$
\begin{aligned}
W_{\rho}\left(\eta, \eta^{k}\right)^{\rho} & \leq \int\left|x-R^{k}(x)\right|^{\rho} \eta(d x)=\int\left|\int y M_{x}(d y)-\int\left(\int z \pi_{y}^{k}(d z)\right) M_{x}(d y)\right|^{\rho} \eta(d x) \\
& \leq \int\left|y-\int z \pi_{y}^{k}(d z)\right|^{\rho} \nu(d y) \leq \iint|y-z|^{\rho} \pi^{k}(d y, d z)=W_{\rho}\left(\nu, \nu^{k}\right)^{\rho},
\end{aligned}
$$

by the martingale property and two applications of Jensen's inequality. The desired conclusion follows.

Remark 6.2. In the context of the previous proposition, if $\eta$ is supported in finitely many atoms, then the condition that $\nu^{k} \rightarrow \nu$ in $\mathcal{W}_{\rho}$ can be relaxed to convergence in $\mathcal{W}_{1}$. To wit, if $\eta=\sum_{i=1}^{\ell} \alpha_{i} \delta_{x^{i}}$, one can take $\rho=1$ in (6.1) and prove

$$
\forall i \leq \ell:\left|x^{i}-R^{k}\left(x^{i}\right)\right| \leq\left(\min \left\{\alpha_{j}\right\}\right)^{-1} W_{1}\left(\nu, \nu^{k}\right),
$$

so taking $\rho$-power and integrating w.r.t. $\eta$ we get $W_{\rho}\left(\eta, \eta^{k}\right)^{\rho} \leq K W_{1}\left(\nu, \nu^{k}\right)^{\rho} \rightarrow 0$.

The previous remark shows that we need to reduce to the finite-support setting. We carry to this in the next two lemmas:

Lemma 6.3. Let $\eta \in \mathcal{P}_{1}\left(\mathbb{R}^{d}\right)$. Then for any $\varepsilon>0$ there is a compactly supported, sub-probability measure $\tilde{\eta}$ with

$$
\tilde{\eta} \leq \eta, \quad \tilde{\eta}\left(\mathbb{R}^{d}\right) \geq 1-\varepsilon, \quad \int_{\mathbb{R}^{d}} z \eta(d z)=\frac{1}{\tilde{\eta}\left(\mathbb{R}^{d}\right)} \int_{\mathbb{R}^{d}} z \tilde{\eta}(d z) .
$$

Proof. We first partition $\mathbb{R}^{d}$ into countably many, disjoint $d$-dimensional cubes $\left(Q_{k}^{\delta}\right)_{k \in \mathbb{N}}$ of length $\delta>0$. Define an approximation $\eta^{\delta}$ of $\eta$ by

$$
\eta^{\delta}:=\sum_{k \in \mathbb{N}} \delta_{z_{k}^{\delta}} \eta\left(Q_{k}^{\delta}\right), \quad z_{k}^{\delta}:= \begin{cases}\frac{1}{\eta\left(Q_{k}^{\delta}\right)} \int_{Q_{k}^{\delta}} z \eta(d z) & \eta\left(Q_{k}^{\delta}\right)>0 \\ 0 & \text { else. }\end{cases}
$$

Note that $\eta^{\delta} \leq_{c} \eta$ and $\eta^{\delta} \rightarrow \eta$ in $\mathcal{W}_{1}$ when $\delta \searrow 0$. If there exists an approximation $\eta^{\delta}$ such that the assertion holds, then it is straightforward to construct the corresponding measure for $\eta$, which in turn satisfies the assertion with respect to $\eta$. W.l.o.g., we may assume that

$$
\left\{\sum_{i=1}^{2 d} \alpha_{i} v_{i}:\left(\alpha_{i}\right)_{i=1}^{2 d} \in \mathbb{R}_{+}^{2 d}, v_{1}, \ldots, v_{2 d} \in\left\{x-\bar{z} \in \mathbb{R}^{d}: x \in \operatorname{supp}(\eta)\right\}\right\}=\mathbb{R}^{d},
$$

where $\bar{z}$ denotes the barycenter of $\eta$. Then we can find $\delta>0$ such that

$$
\left\{\sum_{i=1}^{2 d} \alpha_{i} v_{i}:\left(\alpha_{i}\right)_{i=1}^{2 d} \in \mathbb{R}_{+}^{2 d}, v_{1}, \ldots, v_{2 d} \in\left\{x-\bar{z} \in \mathbb{R}^{d}: x \in \operatorname{supp}\left(\eta^{\delta}\right)\right\}\right\}=\mathbb{R}^{d} .
$$


Let $z_{n_{1}}^{\delta}, \ldots, z_{n_{2 d}}^{\delta}$ span $\mathbb{R}^{d}$ in the sense above and

$$
\eta^{\delta}\left(z_{n_{j}}^{\delta}\right)=\eta\left(Q_{n_{j}}^{\delta}\right)>0 \quad j=1, \ldots, 2 d .
$$

For any $\varepsilon>0$ there is a $\tilde{\varepsilon} \in(0, \varepsilon)$ such that

$$
\left\{\sum_{i=1}^{2 d} \alpha_{i} z_{n_{i}}:\left(\alpha_{i}\right)_{i=1}^{2 d} \in \mathbb{R}_{+}^{2 d}, \sum_{i=1}^{2 d} \alpha_{i}<\varepsilon\right\} \supseteq B_{\tilde{\varepsilon}}(0) .
$$

Besides, there exists a compact set $K \subseteq \mathbb{R}^{d}$ such that

$$
\eta^{\delta}\left(K^{c}\right)<\tilde{\varepsilon}, \quad\left|\bar{z}-\int_{K} z \eta^{\delta}(d z)\right|<\tilde{\varepsilon}
$$

and $z_{n_{1}}^{\delta}, \ldots, z_{n_{2 d}}^{\delta} \in K$. Therefore, we find $\left(\tilde{\alpha}_{i}\right)_{i=1}^{2 d} \in \mathbb{R}_{+}^{2 d}$ with

$$
\bar{z}-\int_{K} z \eta^{\delta}(d z)=\sum_{i=1}^{2 d} \tilde{\alpha}_{i} z_{n_{i}}, \quad \sum_{i=1}^{2 d} \tilde{\alpha}_{i}<\varepsilon .
$$

If $\varepsilon$ is chosen smaller than $\eta^{\delta}\left(z_{n_{i}}^{\delta}\right)$ for all $i=1, \ldots, 2 d$, we can define $\tilde{\eta}^{\delta}$ via

$$
\tilde{\eta}^{\delta}:=\eta^{\delta} \uparrow_{K}-\sum_{i=1}^{2 d} \tilde{\alpha}_{i} \delta_{z_{n_{i}}} .
$$

Lemma 6.4. Let $\mu, \eta \in \mathcal{P}_{\rho}\left(\mathbb{R}^{d}\right)$ and $\theta: \mathbb{R}^{d} \rightarrow \mathbb{R}$ convex satisfying the growth condition (1.8). Then there exists a sequence $\left(\eta^{k}\right)_{k \in \mathbb{N}}$ of finitely supported measures with $\eta^{k} \leq_{c} \eta$, $\eta^{k} \rightarrow \eta$ in $\mathcal{W}_{\rho}$ and $W_{\theta}\left(\mu, \eta^{k}\right) \rightarrow W_{\theta}(\mu, \eta)$.

Proof. For any $\varepsilon>0$ we find a compact set $K_{\varepsilon} \subseteq \operatorname{supp}(\eta)$ such that $\int_{K_{\varepsilon}^{c}}|y|^{\rho} \eta(d y)<\varepsilon$. For any $\delta>0$, the set $K_{\varepsilon}$ can be covered by finitely many, disjoint sets $\left(A_{i}^{\varepsilon, \delta}\right)_{i=1}^{N_{\varepsilon, \delta}}$ with diameter smaller than $\delta$ and $K_{\varepsilon}=\bigcup_{i} A_{i}^{\varepsilon, \delta}$. Define the measure $\eta^{\varepsilon, \delta}$ by

$$
\eta^{\varepsilon, \delta}=\delta_{z_{\varepsilon}} \eta\left(K_{\varepsilon}^{c}\right)+\sum_{i=1}^{N_{\delta}} \delta_{z_{i}^{\varepsilon, \delta}} \eta\left(A_{i}^{\varepsilon, \delta}\right),
$$

where the points $z_{\varepsilon}$ and $\left(z_{i}^{\varepsilon, \delta}\right)_{i=1}^{N_{\varepsilon, \delta}}$ are given by

$$
z_{\varepsilon}:=\frac{1}{\eta\left(K_{\varepsilon}^{c}\right)} \int_{K_{\varepsilon}^{c}} y \eta(d y), \quad z_{i}^{\varepsilon, \delta}:=\frac{1}{\eta\left(A_{i}^{\varepsilon, \delta}\right)} \int_{A_{i}^{\varepsilon, \delta}} y \eta(d y)
$$

By construction, there exists a martingale coupling between $\eta^{\varepsilon, \delta}$ and $\eta$, thus, $\eta^{\varepsilon, \delta} \leq_{c} \eta$. Note that $\theta$ restricted to $K_{\varepsilon}$ is Lipschitz continuous. Then drawing the limit $\delta \rightarrow 0$ yields

$$
\left.\sum_{i=1}^{N_{\varepsilon, \delta}} \delta_{z_{i}^{\varepsilon, \delta}} \eta\left(A_{i}^{\varepsilon, \delta}\right) \rightarrow \eta\right|_{K_{\varepsilon}} \text { in } \mathcal{W}_{\rho}
$$

and by convexity, $\left|z_{\varepsilon}\right|^{\rho} \eta\left(K_{\varepsilon}^{c}\right) \leq \int_{K_{\varepsilon}^{c}}|y|^{\rho} \eta(d y)$. Choosing $\delta(\varepsilon)$ sufficiently small, we have

$$
\mathcal{W}_{\rho}\left(\eta, \eta^{\varepsilon, \delta(\varepsilon)}\right) \leq 2 \varepsilon \text { and } \eta^{\varepsilon, \delta(\varepsilon)} \rightarrow \eta \text { in } \mathcal{W}_{\rho} .
$$

By the growth condition (1.8) we obtain $W_{\theta}\left(\mu, \eta^{\varepsilon, \delta(\varepsilon)}\right) \rightarrow W_{\theta}(\mu, \eta)$.

We can now prove a version of Proposition 6.1 under weaker assumptions:

Lemma 6.5. Let $\left(\nu^{k}\right)_{k \in \mathbb{N}}$ be a sequence in $\mathcal{P}_{1}\left(\mathbb{R}^{d}\right)$ and let $\left(\mu^{k}\right)_{k \in \mathbb{N}}$ be a sequence $\mathcal{P}_{\rho}\left(\mathbb{R}^{d}\right)$ with $\nu^{k} \rightarrow \nu$ in $\mathcal{W}_{1}, \mu^{k} \rightarrow \mu$ in $\mathcal{W}_{\rho}$, where $\rho \geq 1$, and let $\theta: \mathbb{R}^{d} \rightarrow \mathbb{R}$ be a convex functions satisfying the growth constraint (1.8). Then for any $\eta \leq_{c} \nu$ we find a sequence of $\eta^{k} \leq_{c} \nu^{k}$ such that $W_{\theta}\left(\mu^{k}, \eta^{k}\right) \rightarrow W_{\theta}(\mu, \eta)$ and $\eta^{k} \rightarrow \eta$ in $\mathcal{W}_{1}$.

Proof. W.l.o.g. assume that $\theta$ is positive. By Lemma 6.3, we can find for any $\varepsilon>0$ a compactly supported $\hat{\eta}=\tilde{\eta}+\left(1-\tilde{\eta}\left(\mathbb{R}^{d}\right)\right) \delta_{\bar{z}} \in \mathcal{P}_{\rho}\left(\mathbb{R}^{d}\right)$ with $\hat{\eta} \leq_{c} \eta, \mathcal{W}_{1}(\hat{\eta}, \eta)<\varepsilon$ and

$$
W_{\theta}(\mu, \hat{\eta}) \leq W_{\theta}(\mu, \eta)+c \int_{\mathbb{R}^{d}} 1+|x-\bar{z}|^{\rho} \mu(d x)<\infty,
$$

where $\bar{z}:=\int_{\mathbb{R}^{d}} y \eta(d y)$. Using stability of classical optimal transport, see [23, Theorem 5.20], we may assume that $\left|W_{\theta}(\mu, \hat{\eta})-W_{\theta}(\mu, \eta)\right|<\varepsilon$. By Lemma 6.4 we may reduce to the case of finitely supported $\hat{\eta}$. We conclude the proof with Remark 6.2. 
Finally we can give the pending proof of Theorem 1.5:

Proof of Theorem 1.5. Lower-semicontinuity of the map $(\mu, \nu) \mapsto V_{\theta}(\mu, \nu)$ follows from [7, Theorem 1.3]. By [7, Lemma 6.1] we have

$$
V_{\theta}(\mu, \nu)=\inf _{\eta \leq_{c} \nu} W_{\theta}(\mu, \eta),
$$

where the infimum is attained for a measure $\eta \leq_{c} \nu$. By Lemma 6.5 we find a sequence $\eta^{k} \leq_{c} \nu^{k}$, so that again using [7, Lemma 6.1] we have

$$
V_{\theta}(\mu, \nu)=W_{\theta}(\mu, \eta)=\lim _{k} W_{\theta}\left(\mu^{k}, \eta^{k}\right) \geq \lim \sup _{k} V_{\theta}\left(\mu^{k}, \nu^{k}\right) .
$$

If $\theta$ is strictly convex, the infimum in (6.3) is attained by a unique probability measure $\eta^{k} \leq_{c} \nu^{k}, 5$ which in turn is the push-forward of $\mu^{k}$ under a $\mu^{k}$-uniquely defined map $T^{k}$. Moreover, the $W_{\theta}$-optimal transport plan $\pi^{k} \in \Pi\left(\mu^{k}, \nu^{k}\right)$ is uniquely determined by $\mu^{k}(d x) \delta_{T^{k}(x)}(d y):$ Suppose the contrary and let $T^{\prime}(x):=\int_{\mathbb{R}^{d}} y \pi_{x}^{k}(d y)$, then $T^{\prime}\left(\mu^{k}\right) \leq_{c} \nu^{k}$ and we find the contradiction

$$
V_{\theta}\left(\mu^{k}, \nu^{k}\right) \leq \int_{\mathbb{R}^{d}} \theta\left(x-T^{\prime}(x)\right) \mu^{k}(d x)<\int_{\mathbb{R}^{d} \times \mathbb{R}^{d}} \theta(x-y) \pi^{k}(d x, d y)=V_{\theta}\left(\mu^{k}, \nu^{k}\right) .
$$

Hence, by convergence of the values of $V_{\theta}$ and tightness of $\left(\eta_{k}\right)_{k \in \mathbb{N}}$, we deduce the convergence of the $\eta^{k}$ to the optimal $\eta \leq_{c} \nu$ in $\mathcal{W}_{1}$. Suppose that $\mu^{k}=\mu$ for all $k \in \mathbb{N}$, then due to the uniqueness of the optimal transport maps $T$ between $T$ and $T(\mu)$, we can apply Theorem [23, Corollary 5.23] and obtain convergence of the transport maps $T^{k}$ to $T$.

\section{References}

[1] B. Acciaio, J. Backhoff-Veraguas, and A. Zalashko. Causal optimal transport and its links to enlargement of filtrations and continuous-time stochastic optimization. Forthcoming at Stoch. Processes and their Applications, 2019.

[2] A. Alfonsi, J. Corbetta, and B. Jourdain. Sampling of probability measures in the convex order by Wasserstein projection. ArXiv e-prints arXiv:1 709.05287v3, Feb. 2019.

[3] J.-J. Alibert, G. Bouchitte, and T. Champion. A new class of cost for optimal transport planning. hal-preprint, 2018. MR-4028478

[4] J. Backhoff-Veraguas, D. Bartl, M. Beiglböck, and M. Eder. Adapted wasserstein distances and stability in mathematical finance. Forthcoming at Finance and Stochastics, 2019.

[5] J. Backhoff-Veraguas, M. Beiglböck, M. Huesmann, and S. Källblad. Martingale BenamouBrenier: a probabilistic perspective. Forthcoming at Annals of Probability, Aug. 2017.

[6] J. Backhoff-Veraguas, M. Beiglböck, Y. Lin, and A. Zalashko. Causal transport in discrete time and applications. SIAM Journal on Optimization, 27(4):2528-2562, 2017. MR-3738324

[7] J. Backhoff-Veraguas, M. Beiglböck, and G. Pammer. Existence, duality, and cyclical monotonicity for weak transport costs. Forthcoming at Calc. Variations and Partial Diff. Eq., 2019. MR-4029731

[8] M. Beiglböck and N. Juillet. On a problem of optimal transport under marginal martingale constraints. Ann. Probab., 44(1):42-106, 2016. MR-3456332

[9] M. Beiglböck and N. Juillet. Shadow couplings. ArXiv e-prints, Sept. 2016.

[10] M. Fathi and Y. Shu. Curvature and transport inequalities for Markov chains in discrete spaces. Bernoulli, 24(1):672-698, 2018. MR-3706773

[11] W. Gangbo and R. McCann. The geometry of optimal transportation. Acta Math., 177(2):113161, 1996. MR-1440931

[12] N. Gozlan and N. Juillet. On a mixture of brenier and strassen theorems. arXiv preprint arXiv:1808.02681, 2018. MR-4008375

\footnotetext{
${ }^{5}$ The uniqueness of $\eta^{k}, T^{k}$ and $\pi^{k}$ was already shown in [2, Theorem 2.1] for $|\cdot|^{\rho}, \rho>1$
} 
Weak monotone rearrangement on the line

[13] N. Gozlan, C. Roberto, P.-M. Samson, Y. Shu, and P. Tetali. Characterization of a class of weak transport-entropy inequalities on the line. Ann. Inst. Henri Poincaré Probab. Stat., 54(3):1667-1693, 2018. MR-3825894

[14] N. Gozlan, C. Roberto, P.-M. Samson, and P. Tetali. Kantorovich duality for general transport costs and applications. J. Funct. Anal., 273(11):3327-3405, 2017. MR-3706606

[15] K. Marton. A measure concentration inequality for contracting markov chains. Geometric \& Functional Analysis GAFA, 6(3):556-571, 1996. MR-1392329

[16] K. Marton et al. Bounding $\bar{d}$-distance by informational divergence: A method to prove measure concentration. The Annals of Probability, 24(2):857-866, 1996. MR-1404531

[17] P.-M. Samson. Transport-entropy inequalities on locally acting groups of permutations. Electron. J. Probab., 22:Paper No. 62, 33, 2017. MR-3690287

[18] M. Shaked and J. G. Shanthikumar. Stochastic orders. Springer Science \& Business Media, 2007. MR-2265633

[19] Y. Shu. From hopf-lax formula to optimal weak transfer plan. arXiv preprint arXiv:1609.03405, 2016.

[20] Y. Shu. Hamilton-Jacobi equations on graph and applications. Potential Anal., 48(2):125-157, 2018. MR-3748388

[21] M. Talagrand. Concentration of measure and isoperimetric inequalities in product spaces. Publications Mathématiques de l'Institut des Hautes Etudes Scientifiques, 81(1):73-205, 1995. MR-1361756

[22] M. Talagrand. New concentration inequalities in product spaces. Inventiones mathematicae, 126(3):505-563, 1996. MR-1419006

[23] C. Villani. Optimal Transport. Old and New, volume 338 of Grundlehren der mathematischen Wissenschaften. Springer, 2009. MR-2459454 